Universidad Católica del Norte

Antofagasta - Chile

\title{
On a Fuzzy Metric Space and Fuzzy Convergence
}

\author{
Alias B. Khalaf \\ University of Duhok, Iraq \\ and \\ Maha Waleed \\ University of Duhok, Iraq \\ Received : February 2020. Accepted : April 2021
}

\begin{abstract}
In this paper we introduce a new type of fuzzy metric spaces and obtain several properties of it. Also some topological properties and boundedness are investigated. The notion of convergence of fuzzy sequences together with the notion of fuzzy Cauchy sequences in a fuzzy metric are discussed and some basic results related to these notions are investigated.
\end{abstract}

2010 Mathematics Subject Classification: 47H10, 54H25.

Keywords: fuzzy metric, fuzzy convergence, fuzzy Cauchy sequence. 


\section{Introduction}

The fuzzy set theory and applications has established as one of the most active areas of research in many branches of mathematics and engineering. This theory was introduced by Zadeh [20] in 1965 and since then a large number of research papers have published by using the notion of fuzzy sets, fuzzy numbers and fuzzification of several classical theories has also been introduced. Many authors have introduced the concept of fuzzy metric spaces in different ways and also they studied the relation with fuzzy topology. Fuzzy metric spaces usually are introduced by means of the points in the crisp set $X$ with fuzzy distance mapping or by using the fuzzy points in $I^{X}$ with a fuzzy distance between fuzzy points. In 1982 Z. Deng [7], introduced the concept of fuzzy pseudo metric space by using fuzzy points also he introduced the concept of fuzzy topology and defined fuzzy interior and fuzzy cluster points. A. George and P. Veeramani [9] in 1994, introduced a fuzzy metric space by considering points in the crisp set and a fuzzy distance between them. They gave several properties of these spaces also many topological notions are discussed. In 2009 M. Aphane [2], gave some results on fuzzy metric spaces. In [15], [16] certain types of fuzzy sets are introduced in fuzzy topological spaces, while in [14], [17] and [19], some types of continuity of maps are studied in fuzzy topological spaces. In [13] and [18], some types of fuzzy sequences and fuzzy Cauchy sequences are studied in certain fuzzy topological spaces. In metric spaces, the notion of a sequence is a good tool to study important topological properties such as the closure of a set and continuity of maps can be characterized using convergent sequences. Several types of fuzzy convergent and fuzzy Cauchy sequences in a fuzzy metric space are studied in [1],[2], [6], [7], [9] and [10]. Fuzzy boundedness in fuzzy metric spaces is investigated in [21]. In [9], the relation between fuzzy compactness [4] and some types of fuzzy boundedness in fuzzy metric spaces is investigated.

In this paper, we introduce a fuzzy metric space by considering fuzzy points in the family of all fuzzy sets with crisp set $X$. We investigate the concept of fuzzy convergence and we define some types of Fuzzy boundedness in fuzzy metric spaces. The concept of complete fuzzy metric space is introduced and some relation among fuzzy convergence, F-boundedness and fuzzy compactness are investigated. 


\section{Definitions and preliminaries}

By $R, N$ we denote the set of real and natural numbers respectively. All linear spaces are assumed to be over $R$. To make this paper as self-contained as possible, we recall some definitions and results which are needed in the next section.

Definition 2.1. ([20]) Let $X$ be a non-empty set and and $I=[0,1]$, then a fuzzy set $A$ in $X$ is characterized by a membership function $\mu_{A}(x)$ from $X$ to the unit interval $I=[0,1]$. The family of all fuzzy sets on $X$ is denoted by $I^{X}$. It is obvious that $\left|\mu_{A}(x)\right| \leq 1$ and hence every fuzzy set is bounded. We say that a fuzzy set $A$ is empty if $\mu_{A}(x)=0$ for all $x \in X$, the fuzzy empty set is denoted by $\tilde{0}$. The whole fuzzy set has the membership function $\mu_{X}(x)=1$ for all $x \in X$ it is denoted by $\tilde{1}$.

Definition 2.2. ([20]) The union and intersection of two fuzzy sets $A$ and $B$ with membership functions $\mu_{A}(x)$ and $\mu_{B}(x)$ are denoted respectively by $A \vee B$ and $A \wedge B$ and their membership functions are defined as:

$\mu_{(A \vee B)}(x)=\max .\left\{\mu_{A}(x), \mu_{B}(x): x \in X\right\}$.

$\mu_{(A \wedge B)}(x)=\min .\left\{\mu_{A}(x), \mu_{B}(x): x \in X\right\}$.

Definition 2.3. ([20]) Two fuzzy sets $A$ and $B$ are called disjoint if and only if $A \wedge B=\tilde{0}$.

Definition 2.4. [7] A fuzzy point $\lambda$ or $\lambda_{x}^{\alpha}$ in $I^{X}$ is a fuzzy set with membership function defined as

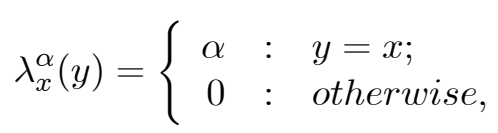

where $0<\alpha<1$. The fuzzy point $\lambda$ is said to have support $x$ and value $\alpha$.

Definition 2.5. [7] The complementary of a fuzzy point $\lambda$ or $\lambda_{x}^{\alpha}$ in $I^{X}$ with support $x$ and value $\alpha$ is a fuzzy point denoted by $\lambda^{c}$ with the same support $x$ and value $1-\alpha$, so

$$
\lambda^{c}=\left(\lambda_{x}^{\alpha}(y)\right)^{c}=\left\{\begin{array}{rll}
1-\alpha & : & y=x \\
0 & : & \text { otherwise. }
\end{array}\right.
$$

The support of $\lambda^{c}$ is $x$ and the value $1-\alpha$. 
We say that two fuzzy points in $I^{X}$ are distinct if either their supports or their values are distinct.

Definition 2.6. [7] A fuzzy point $\lambda$ in $I^{X}$ with support $x$ and value $\alpha$ is called belongs to the fuzzy set $A$ or the fuzzy set $A$ contains $\lambda$ denoted by $\lambda \in A$ if and only if $\lambda(x) \leq \mu_{A}(x)$ i.e. $\alpha \leq \mu_{A}(x)$.

Definition 2.7. [11] A topological space $X$ is called Hausdorff space if and only if for each two distinct points of $X$ there exist two disjoint open sets containing them.

Lemma 2.8. [11] Every metric space is a Hausdorff space.

Definition 2.9. [11] A subset $A$ of a topological space $X$ is called dense if and only if for each open set in $X$ has a non-empty intersection with $A$.

Definition 2.10. [3] A fuzzy topology is a family $\mathcal{F}$ of fuzzy sets in $X$ which satisfies the following conditions:

1. $\tilde{0}, \tilde{1} \in \mathcal{F}$,

2. If $A, B \in \mathcal{F}$, then $A \wedge B \in \mathcal{F}$,

3. If $D$ is any index set and $A_{d} \in \mathcal{F}$ for each $d \in D$, then

$$
\bigcup_{d \in D} A_{d} \in \mathcal{F}
$$

Every member of $\mathcal{F}$ is called a fuzzy open set.

Definition 2.11. [12] A fuzzy topological space $I^{X}$ is called fuzzy Hausdorff space if and only if for each two distinct fuzzy points of $I^{X}$ there exist two disjoint fuzzy open sets containing them.

Definition 2.12. [9] A binary operation $*:[0,1] \times[0,1] \rightarrow[0,1]$ is a continuous $t$-norm if it satisfies the following conditions:

1. $*$ is associative and commutative;

2. * is continuous;

3. $a * 1=a$ for all $a \in[0,1]$; 
4. $a * b \leq c * d$ whenever $a \leq c$ and $b \leq d$, and $a, b, c, d \in[0,1]$.

Obviously, $a * b=a \times b$ and $a * b=\min .\{a, b\}$ are two common examples of continuous $t$-norms.

Definition 2.13. [9] Let $X$ be an arbitrary set. A fuzzy subset $M$ of $X \times X \times[0, \infty]$ is called a fuzzy metric on $X$ if it satisfies the following conditions for all $x, y \in X$ and $t \in R$.

(1) If $t \leq 0$, then $M(x, y, t)=0$;

(2) For all $t>0, M(x, y, t)=1$ if and only if $x=y$;

(3) For all $t>0, M(x, y, t)=M(y, x, t)$;

(4) For all $s, t \in R, x, y \in X, M(x, y, s+t) \geq M(x, z, s) * M(y, z, t)$;

(5) $M(x, y, *)$ is a non decreasing function of $R$ and $\lim _{t \rightarrow \infty} M(x, y, t)=1$.

The pair $(X, M, *)$ is called a fuzzy metric space.

Definition 2.14. [9] Let $(X, M,$.$) be a fuzzy metric space and x \in X$. If $0<r<1$ and $t>0$, then a fuzzy open ball $B(x, r, t)$ with center $x$ and radius $r$ is defined as $B(x, r, t)=\{y \in X: M(x, y, t)>1-r\}$.

Definition 2.15. ([1]) Let $(X, M,$.$) be a fuzzy metric space, a sequence$ $\left\langle x_{n}\right\rangle$ in $X$ is fuzzy convergent to $x \in X$. If for each $\varepsilon>0$ and $t>0$, there exists a large number $K$ such that $x_{n} \in B(x, \varepsilon, t)$ for each $n>K$.

Lemma 2.16. [9]

1. In $(X, M,$.$) , every fuzzy open ball is a fuzzy open set.$

2. The fuzzy metric space $(X, M,$.$) is fuzzy Hausdorff.$

\section{Fuzzy Metric on a Fuzzy Space}

In this section we introduce a fuzzy metric on the family of all fuzzy sets defined on a non-empty set and give some properties of it. The fuzzy points are usually denoted by $\lambda_{x}^{\alpha}, \mu_{y}^{\beta}(x, y$ are their supports and $\alpha, \beta$ are their values) and if there is no confusion we just write them $\lambda, \mu$. Let $X$ be a non-empty set and $I$ be the closed unit interval $[0,1]$, so the family of fuzzy sets on $X$ is denoted by $I^{X}$. 
Definition 3.1. A fuzzy subset $M$ of $I^{X} \times I^{X} \times[0, \infty]$ is called a fuzzy metric on $I^{X}$ if the following conditions are satisfied for all $\lambda_{x}^{\alpha}, \mu_{y}^{\beta} \in I^{X}$ and $t \in[0, \infty]$.

(F1) $M(\lambda, \mu, 0)=0$;

(F2) For all $t>0, M(\lambda, \mu, t)=1$, if and only if $x=y$ and $\alpha \leq \beta$;

(F3) For all $t>0, M(\lambda, \mu, t)=M\left(\mu^{c}, \lambda^{c}, t\right)$;

(F4) For all $s, t \in[0, \infty), \lambda, \mu \in I^{X}, M(\lambda, \mu, s+t) \geq M(\lambda, \eta, s) * M(\eta, \mu, t)$;

(F5) $M(\lambda, \mu, *)$ is a continuous function of $[0, \infty]$ and $\lim _{t \rightarrow \infty} M(\lambda, \mu, t)=1$.

The pair $(X, M)$ is called a fuzzy metric space.

Proposition 3.2. $M(\lambda, \mu, *)$ is a non decreasing function on $[0, \infty]$ for every $\lambda, \mu \in I^{X}$.

Proof. Let $0<s<t$, then by (F4), we have $M(\lambda, \lambda, t-s) * M(\lambda, \mu, s) \leq$ $M(\lambda, \mu, t)$ and by (F2), $M(\lambda, \lambda, t-s)=1$. Hence, $M(\lambda, \mu, s) \leq M(\lambda, \mu, t)$ whenever $a * b$ is either $a \times b$ or $\min .\{a, b\}$.

Remark 3.3. If $\lambda=\lambda_{x}^{\alpha}, \mu=\mu_{y}^{\beta} \in I^{X}$ and $t \in[0, \infty]$, then $M(\lambda, \mu, t)$ is defined to be the degree (probability) of fuzzy closeness between $\lambda$ and $\mu$ with respect to $t \geq 0$. If we use the notation $\tilde{d}(\lambda, \mu)$ the fuzzy distance between $\lambda$ and $\mu$, then

$M(\lambda, \mu, t)=c$ if and only if $P(\tilde{d}(\lambda, \mu) \leq t)=c$ where $P$ is the probability function.

In other words,

$P(\tilde{d}(\lambda, \mu) \leq t)=c$ and $P(\tilde{d}(\mu, \gamma) \leq s)=c$ implies that $P(\tilde{d}(\lambda, \gamma) \leq t+s)=$ $c$.

Or

$M(\lambda, \mu, t) \geq c$ and $M(\mu, \gamma, t) \geq c$ implies that $M(\lambda, \gamma, t+s) \geq c$.

We have $x=y$ and $\alpha \leq \beta$ if and only if $M(\lambda, \mu, t)=1$ for all $t>0$, and

$$
\lim _{t \rightarrow 0} M(\lambda, \mu, t)=0 .
$$


Remark 3.4. Let $\left(I^{X}, M, *\right)$ be a fuzzy metric space and let $\lambda, \mu \in I^{X}$, $t>0,0<c<1$. If $M(\lambda, \mu, t)>1-c$, then for $t_{0}$ with $0<t_{0}<t$ we have $M\left(\lambda, \mu, t_{0}\right)>1-c$.

The following are examples of a fuzzy metric space on the set $X=R$ with $t$-norm $a * b=a \times b$.

Example 3.5. Let $X=R$ and $\lambda_{x_{1}}^{\alpha_{1}}$ and $\mu_{x_{2}}^{\alpha_{2}}$ be any two fuzzy points where $x_{1}, x_{2} \in R$ and $\alpha_{1}, \alpha_{2} \in(0,1]$ and $t \in[0, \infty)$. Then

$$
M(\lambda, \mu, t)=\left\{\begin{array}{rll}
\frac{t}{t+\left(\alpha_{1}-\alpha_{2}\right)+\left|x_{1}-x_{2}\right|} & : & \alpha_{1} \geq \alpha_{2} ; \\
\frac{t}{t+\left|x_{1}-x_{2}\right|} & : & \alpha_{1} \leq \alpha_{2},
\end{array}\right.
$$

is a fuzzy metric.

(F1) is clear.

(F2) Suppose that $M(\lambda, \mu, t)=1$, if $\alpha_{1} \geq \alpha_{2}$, then $\frac{t}{t+\left(\alpha_{1}-\alpha_{2}\right)+\left|x_{1}-x_{2}\right|}=1$. Hence, $\left(\alpha_{1}-\alpha_{2}\right)+\left|x_{1}-x_{2}\right|=0$. Which implies that $\alpha_{1}=\alpha_{2}$ and $x_{1}=x_{2}$.

If $\alpha_{1} \leq \alpha_{2}$, then $\frac{t}{t+\left|x_{1}-x_{2}\right|}=1$ which implies $x_{1}=x_{2}$. For the converse part, If $\alpha_{1} \leq \alpha_{2}$ and $x_{1}=x_{2}$, it is clear that $M(\lambda, \mu, t)=1$.

(F3) Case (1) If $\alpha_{1} \geq \alpha_{2}$, Then $M(\lambda, \mu, t)=\frac{t}{t+\left(\alpha_{1}-\alpha_{2}\right)+\left|x_{1}-x_{2}\right|}$ and $1-\alpha_{2} \geq$ $1-\alpha_{1}$. Hence

$M\left(\mu^{c}, \lambda^{c}, t\right)=\frac{t}{t+\left(1-\alpha_{2}-\left(1-\alpha_{1}\right)\right)+\left|x_{1}-x_{2}\right|}=\frac{t}{t+\left(\alpha_{1}-\alpha_{2}\right)+\left|x_{1}-x_{2}\right|}=M(\lambda, \mu, t)$.

Case(2) if $\alpha_{1} \leq \alpha_{2}$, then $1-\alpha_{2} \leq 1-\alpha_{1}$ and hence

$M(\lambda, \mu, t)=M\left(\mu^{c}, \lambda^{c}, t\right)=\frac{t}{t+\left|x_{1}-x_{2}\right|}$.

(F4) Let $\eta_{x_{3}}^{\alpha_{3}}$ be another fuzzy point. We have the following cases:

(i) If $\alpha_{1} \geq \alpha_{2} \geq \alpha_{3}$, then we have $M(\lambda, \mu, t+s)=\frac{t+s}{t+s+\left(\alpha_{1}-\alpha_{2}\right)+\left|x_{1}-x_{2}\right|}$, $M(\lambda, \eta, t)=\frac{t}{t+\left(\alpha_{1}-\alpha_{3}\right)+\left|x_{1}-x_{3}\right|}$ and $M(\eta, \mu, s)=\frac{s}{s+\left|x_{3}-x_{2}\right|}$. Now we have

$\frac{t+s}{t+s+\left(\alpha_{1}-\alpha_{2}\right)+\left|x_{1}-x_{2}\right|}-\frac{t}{t+\left(\alpha_{1}-\alpha_{3}\right)+\left|x_{1}-x_{3}\right|} \times \frac{s}{s+\left|x_{3}-x_{2}\right|}=$

$\frac{(t+s)\left(t+\left(\alpha_{1}-\alpha_{3}\right)+\left|x_{1}-x_{3}\right|\right)\left(s+\left|x_{3}-x_{2}\right|\right)-t s\left(t+s+\left(\alpha_{1}-\alpha_{2}\right)+\left|x_{1}-x_{2}\right|\right)}{\left(t+s+\left(\alpha_{1}-\alpha_{2}\right)+\left|x_{1}-x_{2}\right|\right)\left(t+\left(\alpha_{1}-\alpha_{3}\right)+\left|x_{1}-x_{3}\right|\right)\left(s+\left|x_{3}-x_{2}\right|\right)}$. We have

$\left|x_{1}-x_{2}\right| \leq\left|x_{1}-x_{3}\right|+\left|x_{3}-x_{2}\right|$, so $-s t\left(\left|x_{1}-x_{2}\right|\right) \geq-s t\left|x_{1}-x_{3}\right|-s t \mid x_{3}-$

$x_{2} \mid$. Also $\alpha_{1}-\alpha_{2} \leq \alpha_{1}-\alpha_{3}$ and hence $-t s\left(\alpha_{1}-\alpha_{2}\right) \geq-t s\left(\alpha_{1}-\alpha_{3}\right)$.

Therefore, we obtain that

$(t+s)\left(t+\left(\alpha_{1}-\alpha_{3}\right)+\left|x_{1}-x_{3}\right|\right)\left(s+\left|x_{3}-x_{2}\right|\right)-t s\left(t+s+\left(\alpha_{1}-\alpha_{2}\right)+\right.$ $\left.\left|x_{1}-x_{2}\right|\right) \geq(t+s)\left(t+\left(\alpha_{1}-\alpha_{3}\right)+\left|x_{1}-x_{3}\right|\right)\left(s+\left|x_{3}-x_{2}\right|\right)-t^{2} s-$ $t s^{2}-t s\left(\alpha_{1}-\alpha_{3}\right)-s t\left|x_{1}-x_{3}\right|-s t\left|x_{3}-x_{2}\right| \geq t^{2}\left(\left|x_{3}-x_{2}\right|\right)+t\left(\alpha_{1}-\right.$ 
$\left.\alpha_{2}\right)\left(\left|x_{3}-x_{2}\right|\right)+s\left(\alpha_{1}-\alpha_{2}\right)\left(\left|x_{3}-x_{2}\right|\right)+s^{2}\left(\alpha_{1}-\alpha_{2}\right)+t\left(\left|x_{3}-x_{2}\right|\right)\left(\mid x_{1}-\right.$ $\left.x_{3} \mid\right)+s^{2}\left(\left|x_{1}-x_{3}\right|\right)+s\left(\left|x_{3}-x_{2}\right|\right)\left(\left|x_{1}-x_{3}\right|\right) \geq 0$. Which implies that $M(\lambda, \mu, s+t) \geq M(\lambda, \eta, s) \times M(\eta, \mu, t)$.

(ii) If $\alpha_{2} \geq \alpha_{1} \geq \alpha_{3}$. Then we have $M(\lambda, \mu, s+t)-M(\lambda, \eta, s) \times$ $M(\eta, \mu, t)=\frac{t+s}{t+s+\left|x_{1}-x_{2}\right|}-\frac{t}{t+\left(\alpha_{1}-\alpha_{3}\right)+\left|x_{1}-x_{3}\right|} \times \frac{s}{s+\left|x_{3}-x_{2}\right|}$ $=\frac{(t+s)\left(t+\left(\alpha_{1}-\alpha_{3}\right)+\left|x_{1}-x_{3}\right|\right)\left(s+\left|x_{3}-x_{2}\right|\right)-t s\left(t+s+\left|x_{1}-x_{2}\right|\right)}{\left(t+s+\left|x_{1}-x_{2}\right|\right)\left(t+\left(\alpha_{1}-\alpha_{3}\right)+\left|x_{1}-x_{3}\right|\right)\left(s+\left|x_{3}-x_{2}\right|\right)} \geq 0$. Hence $M(\lambda, \mu, s+t) \geq M(\lambda, \eta, s) \times M(\eta, \mu, t)$.

(iii) If $\alpha_{2} \geq \alpha_{3} \geq \alpha_{1}$. Then we have $M(\lambda, \mu, s+t)-M(\lambda, \eta, s) \times$ $M(\eta, \mu, t)=\frac{t+s}{t+s+\left|x_{1}-x_{2}\right|}-\frac{t}{t+\left|x_{1}-x_{3}\right|} \times \frac{s}{s+\left|x_{3}-x_{2}\right|}$ $=\frac{(t+s)\left(t+\left|x_{1}-x_{3}\right|\right)\left(s+\left|x_{3}-x_{2}\right|\right)-t s\left(t+s+\left|x_{1}-x_{2}\right|\right)}{\left(t+s+\left|x_{1}-x_{2}\right|\right)\left(t+\left|x_{1}-x_{3}\right|\right)\left(s+\left|x_{3}-x_{2}\right|\right)} \geq 0$. Hence $M(\lambda, \mu, s+t) \geq M(\lambda, \eta, s) \times M(\eta, \mu, t)$.

The other cases are similar.

Example 3.6. Let $X=R$ and $\lambda_{x}^{\alpha}$ and $\mu_{y}^{\beta}$ be any two fuzzy points where $x, y \in R, \alpha, \beta \in(0,1]$ and $t \geq 0$. Then

$$
M(\lambda, \mu, t)=\left\{\begin{aligned}
e^{-\frac{|x-y|+(\alpha-\beta)}{t}} & : \quad \alpha \geq \beta ; \\
e^{-\frac{|x-y|}{t}} & : \quad \alpha \leq \beta,
\end{aligned}\right.
$$

is a fuzzy metric.

(F1) It is clear that $M(\lambda, \mu, 0)=0$.

(F2) Suppose that for $t>0, M(\lambda, \mu, t)=1$.

If $\alpha \geq \beta$, then we have $e^{-\frac{|x-y|+(\alpha-\beta)}{t}}=1$ and hence, $\frac{|x-y|+(\alpha-\beta)}{t}=0$ which implies that $|x-y|+(\alpha-\beta)=0$. Therefore, $x=y$ and $\alpha=\beta$. If $\alpha \leq \beta$, we obtain that $x=y$.

Conversely, suppose that $x=y$ and $\alpha \leq \beta$, so $M(\lambda, \mu, t)=e^{0}=1$.

(F3) $M\left(\mu^{c}, \lambda^{c}, t\right)=e^{-\frac{|x-y|+((1-\beta)-(1-\alpha))}{t}}$ when $(1-\beta) \geq(1-\alpha)$. Hence $M\left(\mu^{c}, \lambda^{c}, t\right)=e^{-\frac{|x-y|+(\alpha-\beta)}{t}}$ when $\left.\alpha \geq \beta\right)$. Therefore, $M\left(\mu^{c}, \lambda^{c}, t\right)=$ $M(\lambda, \mu, t)$. Further we have $(1-\beta) \leq(1-\alpha)$ implies that $\alpha \leq \beta$ and hence $M\left(\mu^{c}, \lambda^{c}, t\right)=M(\lambda, \mu, t)$. 
(F4) To prove that $M(\lambda, \mu, s+t) \geq M(\lambda, \eta, s) \times M(\eta, \mu, t)$ where the fuzzy point $\eta=\eta_{z}^{\gamma}$. For each $x, y, z \in R$ and $\left.s, t>\right)$, the following inequality is true:

$|x-y| \leq\left(\frac{t+s}{t}\right)|x-z|+\left(\frac{t+s}{s}\right)|z-y|$. That is

$$
\frac{|x-y|}{t+s} \leq\left(\frac{|x-z|}{s}\right)+\left(\frac{|z-y|}{t}\right) .
$$

For values of $\alpha, \beta$ and $\gamma$, we have the following cases:

(1) If $\alpha \leq \beta, \alpha \leq \gamma$ and $\gamma \leq \beta$, then from Equation 3.1, we obtain that

$$
e^{-\left(\frac{|x-y|}{t+s}\right)} \geq e^{-\left(\frac{|x-z|}{s}\right)} \times e^{-\left(\frac{|z-y|}{t}\right)} .
$$

Hence, $M(\lambda, \mu, s+t) \geq M(\lambda, \eta, s) \times M(\eta, \mu, t)$.

(2) If $\alpha \geq \beta, \alpha \geq \gamma$ and $\gamma \geq \beta$, also we have

$$
\frac{(\alpha-\beta)}{t+s} \leq\left(\frac{(\alpha-\gamma)}{s}\right)+\left(\frac{(\gamma-\beta)}{t}\right) .
$$

Combining Equation 3.1 and Equation 3.2, we get

$$
(3.3) \frac{|x-y|+(\alpha-\beta)}{t+s} \leq\left(\frac{|x-z|+(\alpha-\gamma)}{s}\right)+\left(\frac{|z-y|+(\gamma-\beta)}{t}\right) .
$$

Again we obtain that $M(\lambda, \mu, s+t) \geq M(\lambda, \eta, s) \times M(\eta, \mu, t)$.

(3) If $\gamma \geq \alpha \geq \beta$, then $\alpha-\beta \leq \gamma-\beta$ and hence $\frac{(\alpha-\beta)}{t+s} \leq\left(\frac{(\gamma-\beta)}{t}\right)$. Therefore, we get

$$
\frac{|x-y|+(\alpha-\beta)}{t+s} \leq\left(\frac{|x-z|}{s}\right)+\left(\frac{|z-y|+(\gamma-\beta)}{t}\right)
$$

Hence, $M(\lambda, \mu, s+t) \geq M(\lambda, \eta, s) \times M(\eta, \mu, t)$.

The other cases can be proved similarly.

Proposition 3.7. If condition [F3] in Definition 3.1, $M(\lambda, \mu, t)=M\left(\mu^{c}, \lambda^{c}, t\right)$ for all $t \in(0, \infty]$, is replaced by: $[F 3]^{\prime} M(\lambda, \mu, t)=M(\mu, \lambda, t)$ for all $t \geq 0$, then $\left(I^{X}, M, *\right)$ is also a fuzzy metric space where either $a * b=a \times b$ or $a * b=\min .\{a, b\}$. 


\section{Proof.}

(F1) is obvious.

(F2) Let $\lambda_{x}^{\alpha}, \mu_{x}^{\beta}$ be two fuzzy numbers with $\alpha \leq \beta$, then from condition (F2) and by hypothesis, we have $M\left(\lambda_{x}^{\alpha}, \mu_{x}^{\beta}, t\right)=M\left(\mu_{x}^{\beta}, \lambda_{x}^{\alpha}, t\right)=1$.

(F3) Let $\lambda=\lambda_{x}^{\alpha}, \lambda^{\prime}=\lambda_{x}^{\alpha^{\prime}}, \alpha \leq \alpha^{\prime}$ and $\mu=\mu_{y}^{\beta}, \mu^{\prime}=\mu_{y}^{\beta^{\prime}}$ and $x \neq y$. If $\beta \leq \beta^{\prime}$, then $M(\lambda, \mu, s+t)=M(\mu, \lambda, s+t) \geq M\left(\mu, \mu^{\prime}, s\right) *$ $M\left(\mu^{\prime}, \lambda, t\right)=M\left(\mu^{\prime}, \lambda, t\right)=M\left(\lambda, \mu^{\prime}, t\right)$. Hence, by Remark 3.4, $M(\lambda, \mu, t) \geq$ $M\left(\lambda, \mu^{\prime}, t\right)$. Also we have $M\left(\lambda, \mu^{\prime}, t+s\right) \geq M(\lambda, \mu, t) * M\left(\mu, \mu^{\prime}, s\right)=$ $M(\lambda, \mu, t)$ and again by Remark 3.4 $M\left(\lambda, \mu^{\prime}, t\right) \geq M(\lambda, \mu, t)$. Therefore, $M(\lambda, \mu, t)=M\left(\lambda, \mu^{\prime}, t\right)$. By the same way we can prove that $M\left(\lambda, \mu^{\prime}, t\right)=M\left(\lambda^{\prime}, \mu^{\prime}, t\right)$ and hence, we obtain that $M(\lambda, \mu, t)=$ $M\left(\lambda^{\prime}, \mu^{\prime}, t\right)$.

If $\beta \geq \beta^{\prime}$, from above we have $M\left(\lambda, \mu^{\prime}, t\right)=M\left(\lambda^{\prime}, \mu, t\right)$. Also we have $M\left(\lambda^{\prime}, \mu^{\prime}, s+t\right)=M\left(\mu^{\prime}, \lambda^{\prime}, s+t\right) \geq M\left(\mu^{\prime}, \mu, s\right) * M\left(\mu, \lambda^{\prime}, t\right)=M\left(\mu, \lambda^{\prime}, t\right)=$ $M\left(\lambda^{\prime}, \mu, t\right)=M\left(\lambda, \mu^{\prime}, t\right)$. Again, we have $M\left(\lambda, \mu^{\prime}, s+t\right) \geq M\left(\lambda, \lambda^{\prime}, s\right) * M\left(\lambda^{\prime}, \mu^{\prime}, t\right)=M\left(\lambda^{\prime}, \mu^{\prime}, t\right)$.

Therefore, $M\left(\lambda^{\prime}, \mu^{\prime}, t\right)=M\left(\lambda, \mu^{\prime}, t\right)$.

In the same way we can prove that $M(\lambda, \mu, t)=M\left(\lambda, \mu^{\prime}, t\right)$ and hence, we get $M(\lambda, \mu, t)=M\left(\lambda^{\prime}, \mu^{\prime}, t\right)=M\left(\mu^{\prime}, \lambda^{\prime}, t\right)$. Now in particular if we take $\beta^{\prime}=1-\beta$ and $\alpha^{\prime}=1-\alpha$, then we obtain that $M(\lambda, \mu, t)=M\left(\mu^{c}, \lambda^{c}, t\right)$.

Proposition 3.8. If $\alpha_{1} \leq \alpha$, then $M\left(\lambda^{\alpha_{1}}, \mu, t\right) \geq M\left(\lambda^{\alpha}, \mu, t\right)$.

Proof. We have by (F4), $M\left(\lambda^{\alpha_{1}}, \mu, s+t\right) \geq \min .\left\{M\left(\lambda^{\alpha_{1}}, \lambda, s\right), M(\lambda, \mu, t)\right\}=$ $M(\lambda, \mu, t)$. By Remark 3.4, we get $M\left(\lambda^{\alpha_{1}}, \mu, t\right) \geq M\left(\lambda^{\alpha}, \mu, t\right)$.

Proposition 3.9. If $M\left(\lambda, \mu^{\beta^{\prime}}, t\right)>r$ for some $0<r<1$ and $\beta^{\prime} \leq \beta$, then $M\left(\lambda, \mu^{\beta}, t\right)>r$.

Proof. If $M\left(\lambda, \mu^{\beta^{\prime}}, t\right)>r$, then by (F3), $M\left(\mu^{\beta^{\prime c}}, \lambda^{c}, t\right)>r$ and by Proposition 3.8, for any $\gamma \leq 1-\beta^{\prime}$ we have $M\left(\mu^{\gamma}, \lambda^{c}, t\right) \geq M\left(\mu^{\beta^{\prime c}}, \lambda^{c}, t\right)>r$ . Therefore, $M\left(\lambda, \mu^{\beta}, t\right)>r$ where $\beta=1-\gamma \geq \beta^{\prime}$.

Proposition 3.10. If $\beta^{\prime} \leq \beta$, then $M\left(\lambda, \mu^{\beta^{\prime}}, t\right) \leq M\left(\lambda, \mu^{\beta}, t\right)$. 
Proof. We have by (F3), $M\left(\lambda, \mu^{\beta^{\prime}}, t\right)=M\left(\mu^{\beta^{\prime c}}, \lambda^{c}, t\right)$ and Proposition 3.8, $M\left(\mu^{\beta^{\prime c}}, \lambda^{c}, t\right) \leq M\left(\mu^{c}, \lambda^{c}, t\right)=M(\lambda, \mu, t)$, where $1-\beta \leq 1-\beta^{\prime}$. Therefore, $M\left(\lambda, \mu^{\beta^{\prime}}, t\right) \leq M\left(\lambda, \mu^{\beta}, t\right)$, where $\beta^{\prime} \leq \beta$.

Remark 3.11. In general condition (F3) does not implies condition $\left(F 3^{\prime}\right)$. However we have the following result:

Proposition 3.12. If $\lambda_{x}^{\alpha}, \mu_{y}^{\beta} \in I^{X}$ and if $\alpha=\beta$. Then $(F 3)$ and $\left(F 3^{\prime}\right)$ are equivalent.

Proof. Suppose that $\lambda_{x}^{\alpha}, \mu_{y}^{\beta} \in I^{X}$ and $\alpha=\beta$. We have the following cases:

Case 1. If $\alpha \leq(1-\beta)$, Then by Proposition 3.8, we get $M\left(\lambda^{\alpha}, \mu, t\right) \geq$ $M\left(\lambda^{1-\beta}, \mu, t\right)=M\left(\lambda^{1-\beta}, \mu, t\right)$

Definition 3.13. Let $\left(I^{X}, M, *\right)$ be a fuzzy metric space and $\lambda \in I^{X}$. If $0<r<1$ and $t>0$, then

1. A fuzzy open ball $B(\lambda, r, t)$ with center $\lambda$ and radius $r$ is defined as $B(\lambda, r, t)=\left\{\mu \in I^{X}: M(\lambda, \mu, t)>1-r\right\}$.

2. A fuzzy closed ball $B[\lambda, r, t]$ with center $\lambda$ and radius $r$ is defined as $B[\lambda, r, t]=\left\{\mu \in I^{X}: M(\lambda, \mu, t) \geq 1-r\right\}$.

Definition 3.14. Let $\left(I^{X}, M, *\right)$ be a fuzzy metric space. A fuzzy set $A$ of $I^{X}$ is said to be fuzzy compact, if for $0<r<1, t>0$ and $A \subseteq \bigcup\{B(\lambda, r, t)$ : $\lambda \in A\}$, then there is a finite set $\left\{\lambda_{1}, \lambda_{2}, \ldots, \lambda_{n}\right\}$ such that

$$
A \subseteq \bigcup_{i=1}^{n}\left\{B\left(\lambda_{i}, r, t\right): \lambda_{i} \in A\right\}
$$

Proposition 3.15. If $B(\lambda, r, t)$ is a soft open ball in the fuzzy metric space $\left(I^{X}, M, *\right)$, then for each $\mu \in B(\lambda, r, t)$, there exists a soft open ball $B(\mu, p, q)$ such that $\mu \in B(\mu, p, q) \subseteq B(\lambda, r, t)$. 
Proof. For $\mu \in B(\lambda, r, t)$, so $M(\lambda, \mu, t)>1-r$. Hence, by Remark 3.4, there exists $0<t_{0}<t$ such that $M\left(\lambda, \mu, t_{0}\right)>1-r$. If $r_{0}=M\left(\lambda, \mu, t_{0}\right)$, then $r_{0}>1-r$, so we can find a number $s$ with $r_{0}>1-s>1-r$ and hence, there exists $r_{1}, 0<r_{1}<1$ such that $r_{0} \times r_{1}>1-s$. Suppose that $p=1-r_{1}$ and $q=t-t_{0}$, if $\eta \in B(\mu, p, q)$ this implies that $M\left(\mu, \eta, t-t_{0}\right)>1-p=$ $r_{1}$. Hence, by (F4), we have $M(\lambda, \eta, t) \geq \min \left\{M\left(\lambda, \mu, t_{0}\right), M\left(\mu, \eta, t-t_{0}\right)\right\}$ implies that $M(\lambda, \eta, t) \geq r_{0} \times r_{1} \geq 1-s>1-r$. Therefore, $\eta \in B(\lambda, r, t)$ and thus $\mu \in B(\mu, p, q) \subseteq B(\lambda, r, t)$.

Proposition 3.16. If the soft metric $\left(I^{X}, M, *\right)$ satisfies $\left(F 3^{\prime}\right)$. Then it is soft Hausdorff.

Proof. Let $\lambda_{x}^{\alpha}, \mu_{y}^{\beta}$ be two distinct fuzzy points in $I^{X}$. Then $0<$ $M(\lambda, \mu, t)<1$ and let $M(\lambda, \mu, t)=r$ where $0<r<1$. Hence, for each $r<$ $r_{0}<1$, there exists $r_{1}$ such that $r_{1} * r_{1} \geq r_{0}$. Consider the fuzzy open balls $B\left(\lambda, 1-r_{1}, \frac{1}{2} t\right)$ and $B\left(\mu, 1-r_{1}, \frac{1}{2} t\right)$. If $\eta \in B\left(\lambda, 1-r_{1}, \frac{1}{2} t\right) \cap B\left(\mu, 1-r_{1}, \frac{1}{2} t\right)$, then $r=M(\lambda, \mu, t) \geq M\left(\lambda, \eta, \frac{1}{2} t\right) * M\left(\eta, \mu, \frac{1}{2} t\right) \geq r_{1} * r_{1} \geq r_{0}>r$ which is contradiction. Hence, $B\left(\lambda, 1-r_{1}, \frac{1}{2} t\right) \cap B\left(\mu, 1-r_{1}, \frac{1}{2} t\right)=\phi$. Therefore, $\left(I^{X}, M, *\right)$ is soft Hausdorff.

\section{The induced fuzzy metric}

In this section we define the fuzzy metric space $(X, M)$ when $(X, d)$ is any metric space and we denote this fuzzy metric by $M_{d}$.

Proposition 4.1. Let $(X, d)$ be a metric space and let $\lambda_{x}^{\alpha}, \mu_{y}^{\beta} \in I^{X}$. Then $M_{d}(\lambda, \mu, t)=\frac{t}{t+d(x, y)+\max \cdot\{0, \alpha-\beta\}}$ is a fuzzy metric on $I^{X}$.

\section{Proof.}

(F1) when $t=0$, then clearly $M_{d}(\lambda, \mu, 0)=0$.

(F2) If $t>0$, suppose that $M_{d}(\lambda, \mu, t)=1$, then $d(x, y)+\max .\{0, \alpha-\beta\}=$ 0 implies that $d(x, y)=0$ and $\max .\{0, \alpha-\beta\}=0$. Hence, $x=y$ and $\alpha \leq \beta$. Conversely is obvious.

(F3) We have, $M_{d}\left(\mu^{c}, \lambda^{c}, t\right)=\frac{t}{t+d(y, x)+\max .\{0,(1-\beta)-(1-\alpha)\}}$

$$
=\frac{t}{t+d(x, y)+\max \cdot\{0, \alpha-\beta\}}=M_{d}(\lambda, \mu, t) \text {. }
$$


(F4) Let $\eta_{z}^{\gamma} \in I^{X}$, then we have:

$$
\begin{aligned}
M_{d}(\lambda, \mu, t+s) & =\frac{t+s}{t+s+d(x, y)+\max \cdot\{0, \alpha-\beta\}} \\
M_{d}(\lambda, \eta, t) & =\frac{t}{t+d(x, z)+\max \cdot\{0, \alpha-\gamma\}} \\
M_{d}(\eta, \mu, s) & =\frac{s}{s+d(z, y)+\max \cdot\{0, \gamma-\beta\}}
\end{aligned}
$$

We shall discus the following cases:

Case 1. If $\alpha \leq \beta \leq \gamma$. Then

$$
\begin{gathered}
M_{d}(\lambda, \mu, t+s)-M_{d}(\lambda, \eta, t) * M_{d}(\eta, \mu, s) \\
=\frac{t+s}{t+s+d(x, y)}-\frac{t}{t+d(x, z)} * \frac{s}{s+d(z, y)+(\gamma-\beta)} \\
=\frac{(t+s)(t+d(x, z))(s+d(z, y)+(\gamma-\beta))-t s(t+s+d(x, y))}{(t+s+d(x, y))(t+d(x, z))(s+d(z, y)+(\gamma-\beta))} \\
\geq \frac{(t+s)(d(x, z))(d(z, y)+t s(d(x, z)+d(x, z))+(\gamma-\beta))-t s(d(x, z)+d(x, z))}{(t+s+d(x, y))(t+d(x, z))(s+d(z, y)+(\gamma-\beta))} \geq 1
\end{gathered}
$$

The other cases can be proved similarly. This proves (F4).

It is obvious that $M_{d}$ is continuous on $[0, \infty]$ and $\lim _{t \rightarrow \infty} M_{d}(\lambda, \mu, t)=1$.

Therefore, $\left(M_{d}, I^{X}\right)$ is a fuzzy metric on $I^{X}$.

Definition 4.2. Let $\left(M, I^{X}\right)$ be a fuzzy metric space.

1. A fuzzy subset $A$ of $I^{X}$ is said to be $F_{1}$-bounded if there exist $t>0$ and $0<r<1$ such that $M(\lambda, \mu, t)>1-r$ for all $\lambda, \mu \in A$.

2. A crisp subset $B$ of $X$ is said to be $F_{2}$-bounded if there exist $t>0$ and $0<r<1$ such that $M\left(\lambda_{x}^{\alpha}, \mu_{y}^{\beta}, t\right)>1-r$ for all $x, y \in B$ and all $\alpha, \beta \in(0,1]$.

Remark 4.3. If $B$ is the set of all supports of points of $A$. Then $B$ is $F_{2}$-bounded if and only if $A$ is $F_{1}$-bounded.

Proposition 4.4. Let $\left(M_{d}, I^{X}\right)$ be the induced fuzzy metric space. Then $A \subseteq I^{X}$ is $F_{1}$-bounded if and only if $B \subseteq X$ is $F_{2}$-bounded. 
Proof. Suppose that $A \subseteq I^{X}$ is $F_{1}$-bounded, then for each $t>0$ and $0<r<1$, we have $M_{d}(\lambda, \mu, t)>1-r$ for all $\lambda, \mu \in A$. Hence, $\frac{t}{t+d(x, y)+\max .\{0, \alpha-\beta\}}>1-r$ if and only if $t-(1-r)[t+d(x, y)+\max .\{0, \alpha-$ $\beta\}]>0$. Hence,

$$
d(x, y)+\max .\{0, \alpha-\beta\}<r[t+d(x, y)+\max .\{0, \alpha-\beta\}] .
$$

Therefore, we obtain that $d(x, y)<d(x, y)+\max .\{0, \alpha-\beta\}<s$, so $B$ is bounded.

Conversely, if $B$ is bounded, then there is a positive number $s$ such that $d(x, y)<s$ for all $x, y \in B$. Since $B$ is the support set of elements of $A$, so we have

$M_{d}(\lambda, \mu, t)=\frac{t}{t+d(x, y)+\max .\{0, \alpha-\beta\}}>\frac{t}{t+s+\max .\{0, \alpha-\beta\}}>1-r$

where $r=\frac{s+\max .\{0, \alpha-\beta\}}{t+s+\max .\{0, \alpha-\beta\}}$. It is clear that $0<r<1$. Therefore, $A$ is $F_{1}$-bounded.

Proposition 4.5. Let $\left(M, I^{X}\right)$ be any fuzzy metric space and let $A \subseteq I^{X}$ be fuzzy compact, then it is $F_{1}$-bounded.

Proof. Since $A$ is fuzzy compact, so for $t>0$ and $0<r<1$, there is a finite set $\left\{\lambda_{1}, \lambda_{2}, \ldots, \lambda_{n}\right\}$ such that

$$
A \subseteq \bigcup_{i=1}^{n}\left\{B\left(\lambda_{i}, r, t\right): \lambda_{i} \in A\right\} .
$$

To show that $A$ is $F_{1}$-bounded, let $\lambda, \mu \in A$, then there exist $j, k$ such that $\lambda \in B\left(\lambda_{j}, r, t\right)$ and $\mu \in B\left(\lambda_{k}, r, t\right)$ for $0<j, k<n$. This implies that $M\left(\lambda_{j}, \lambda, t\right)>1-r$ and $M\left(\lambda_{k}, \mu, t\right)>1-r$. Since $\lambda_{j}, \lambda_{k}$ are distinct for $0<$ $j, k<n$, so for $t>0$, we have $M\left(\lambda_{j}, \lambda_{k}, t\right)>0$. Let $c=\min .\left\{M\left(\lambda_{j}, \lambda_{k}, t\right)\right.$ : $0<j, k<n$ and because $0<r<1$, so we can take $c<\frac{1}{(1-r)^{2}}$, then we have for $t>0$, then $s=3 t>0$ and $M(\lambda, \mu, s) \geq M\left(\lambda, \lambda_{j}, t\right) * M\left(\lambda_{j}, \lambda_{k}, t\right) *$ $M\left(\mu, \lambda_{k}, t\right)>(1-r)(1-r) c>1-p$ where $0<p<1$. Hence $M(\lambda, \mu, s)>$ $1-p$ for all $\lambda, \mu \in A$, hence $A$ is $F_{1}$-bounded.

Definition 4.6. A sequence in $I^{X}$ is a mapping $f: N \rightarrow I^{X}$ and it is denoted by $\left\langle\lambda_{n}>\right.$ or $\left\langle\lambda_{x_{n}}^{\alpha_{n}}>\right.$ where the membership function of $\lambda_{n}$ is given by: 


$$
\mu_{\lambda_{n}}(x)=\left\{\begin{aligned}
\alpha_{n} & : \text { if } x=x_{n} \\
0 & : \text { otherwise }
\end{aligned}\right.
$$

We say that $\left\langle\lambda_{n}>\right.$ is fuzzy convergent to a fuzzy point $\lambda_{x}^{\alpha}$ (dented by $\left.<\lambda_{n}>\rightarrow \lambda_{x}^{\alpha}\right)$ if and only if

$$
\lim _{n \rightarrow \infty} M\left(\lambda, \lambda_{n}, t\right)=1 \quad \forall t>0 .
$$

Proposition 4.7. A fuzzy sequence $<\lambda_{n}>$ in a fuzzy metric space $\left(I^{X}, M, *\right)$ is fuzzy convergent to a fuzzy point $\lambda_{x}^{\alpha}$ if and only if for each small real number $\varepsilon>0$, there exists a number $K \in N$ such that $\lambda_{n} \in B(\lambda, \varepsilon, t)$ for all $n>K$.

Proof. Suppose that $\left\langle\lambda_{n}>\rightarrow \lambda_{x}^{\alpha}\right.$, then by definition

$$
\lim _{n \rightarrow \infty} M\left(\lambda, \lambda_{n}, t\right)=1 \quad \forall t>0 .
$$

$\Longleftrightarrow$ for each small number $\varepsilon>0$, there exists a number $K \in N$ such that

$$
\left|M\left(\lambda, \lambda_{n}, t\right)-1\right|<\varepsilon \text { for all } n>K
$$

$\Longleftrightarrow 1-M\left(\lambda, \lambda_{n}, t\right)<\varepsilon$ for all $n>K$

$\Longleftrightarrow M\left(\lambda, \lambda_{n}, t\right)>1-\varepsilon$

$\Longleftrightarrow \lambda_{n} \in B(\lambda, \varepsilon, t)$ for all $n>K$.

Example 4.8. Let $(X, d)=(R,||$.$\left.) and let I^{R}, M_{|.|}\right)$be the induced fuzzy metric space. If the sequence $\left\langle\lambda_{n}\right\rangle$ has the membership function

$$
\mu_{\lambda_{n}}(x)=\left\{\begin{array}{rll}
\frac{n}{2 n+1} & : \text { if } \quad x_{n}=\frac{1+n}{n} \\
0 & : & \text { otherwise. }
\end{array}\right.
$$

Then $\left\langle\lambda_{n}>\right.$ converges to $\lambda$ where

$$
\mu_{\lambda}(x)=\left\{\begin{array}{lll}
\frac{1}{2} & : & \text { if } \quad x=1 \\
0 & : & \text { otherwise }
\end{array}\right.
$$

To prove it, we have to show that

$$
\lim _{n \rightarrow \infty} M_{|.|}\left(\lambda, \lambda_{n}, t\right)=1 \quad \forall t>0 .
$$


For this we have $M_{|\cdot|}\left(\lambda_{1}^{\frac{1}{2}}, \lambda_{\frac{1+n}{n}}^{\frac{n}{2 n+1}}, t\right)=\frac{t}{t+\left|1-\frac{1+n}{n}\right|+\left(\frac{1}{2}-\frac{n}{2 n+1}\right)}=\frac{t}{t+\left|\frac{1}{n}\right|+\frac{1}{2(2 n+1)}}$. Hence it is obvious that

$$
\lim _{n \rightarrow \infty} M_{|\cdot|}\left(\lambda_{1}^{\frac{1}{2}}, \lambda_{\frac{1+n}{n}}^{\frac{n}{2 n+1}}, t\right)=1 \quad \forall t>0 .
$$

Theorem 4.9. Let $\left(I^{X}, M_{d} *\right)$ be the induced fuzzy metric space. if $<$ $x_{n}>$ converges to $x$ in $(X, d)$ and $\left\langle\alpha_{n}>\right.$ converges to $\alpha$ in $[0,1]$. Then the fuzzy sequence $\left\langle\lambda_{n}>\right.$ in $I^{X}$ fuzzy converges to $\lambda$.

Proof. Let $t>0$ and by hypothesis $\left\langle x_{n}\right\rangle$ converges to $x$ in $(X, d)$ and $\left\langle\alpha_{n}\right\rangle$ converges to $\alpha$ in $[0,1]$. Therefore, there exists $N_{0}$ such that $d\left(x_{n}, x\right)<\varepsilon_{1}$ and $\left|\alpha_{n}-\alpha\right|<\varepsilon_{1}$ for all $n>N_{0}$. Now $d\left(x_{n}, x\right)+$ $\max .\left\{0, \alpha-\alpha_{n}\right\}<\varepsilon_{1}+\varepsilon_{2}$ and hence, $\frac{t}{t+d\left(x_{n}, x\right)+\max .\left\{0, \alpha-\alpha_{n}\right.}>\frac{t}{t+\varepsilon_{1}+\varepsilon_{2}}$. Therefore, $M_{d}\left(\lambda, \lambda_{n}, t\right)>1-\frac{\varepsilon_{1}+\varepsilon_{2}}{t+\varepsilon_{1}+\varepsilon_{2}}$. Thus, $M_{d}\left(\lambda, \lambda_{n}, t\right)>1-r$ where $r=\frac{\varepsilon_{1}+\varepsilon_{2}}{t+\varepsilon_{1}+\varepsilon_{2}}$, clearly $0<r<1$. This implies that $\lambda_{n} \in B(\lambda, r, t)$, so by Proposition 4.7, $<\lambda_{n}>\rightarrow \lambda$.

Theorem 4.10. Let $\left(I^{X}, M_{d}, *\right)$ be the induced fuzzy metric space. if $<x_{n}>$ converges to $x$ in $(X, d)$, then the fuzzy sequence $\left\langle\lambda_{x_{n}}^{\alpha_{n}}>\right.$ in $I^{X}$ fuzzy converges to $\lambda_{x}^{\alpha}$ where $\alpha \leq \alpha_{n}$ for all $n \in N$.

Proof. Let $t>0$ and by hypothesis $\left\langle x_{n}\right\rangle$ converges to $x$ in $(X, d)$. Therefore, there exists $N_{0}$ such that $d\left(x_{n}, x\right)<\varepsilon$ for all $n>N_{0}$. Since $\alpha \leq \alpha_{n}$ for all $n \in N$, then $d\left(x_{n}, x\right)+\max .\left\{0, \alpha-\alpha_{n}\right\}<\varepsilon$ and hence, $\frac{t}{t+d\left(x_{n}, x\right)+\max .\left\{0, \alpha-\alpha_{n}\right.}>\frac{t}{t+\varepsilon}$. Therefore, $M_{d}\left(\lambda, \lambda_{n}, t\right)>1-\frac{\varepsilon}{t+\varepsilon}$. Thus, $M_{d}\left(\lambda, \lambda_{n}, t\right)>1-r$ where $r=\frac{\varepsilon}{t+\varepsilon}$, clearly $0<r<1$. This implies that $\lambda_{n} \in B(\lambda, r, t)$, so by Proposition 4.7, $<\lambda_{n}>\rightarrow \lambda$.

Remark 4.11. In Example 4.8 and by virtue of Theorem 4.10, the fuzzy sequence $\left\langle\lambda_{\frac{1+n}{n}}^{\frac{n}{2 n+1}}>\right.$ fuzzy converges to $<\lambda_{1}^{\frac{1}{3}}>$ also because $\frac{1}{3} \leq \frac{n}{2 n+1}$ for all $n \in N$. Hence, we obtain that $\left\langle\lambda_{1}^{\frac{1}{3}}>\right.$ and $\left\langle\lambda_{1}^{\frac{1}{2}}>\right.$ are two distinct fuzzy points in $I^{X}$ but every fuzzy open ball containing $\left\langle\lambda_{1}^{\frac{1}{3}}>\right.$ intersects with every open ball containing $\left\langle\lambda_{1}^{\frac{1}{2}}>\right.$. This implies that the fuzzy metric space $\left(I^{X}, M_{d}, *\right)$ is not fuzzy Hausdorff. It is obvious that this space does not satisfies the condition in Proposition 3.16. 
Definition 4.12. A sequence $<\lambda_{x_{n}}^{\alpha}>$ in $I^{X}$ is a fuzzy Cauchy sequence if and only if

$$
\lim _{n, m \rightarrow \infty} M\left(\lambda_{x_{n}}^{\alpha}, \lambda_{x_{m}}^{\alpha}, t\right)=1 \quad \forall t>0 .
$$

Equivalently, for each $\varepsilon>0, t>0$, there exists $K \in N$ such that

$$
M\left(\lambda_{x_{n}}^{\alpha}, \lambda_{x_{m}}^{\alpha}, t\right)>1-\varepsilon \quad \forall n, m>K
$$

Definition 4.13. A fuzzy metric space $\left(I^{X}, M, *\right)$ is called fuzzy complete if every fuzzy Cauchy sequence is fuzzy convergent in it.

Theorem 4.14. Let $\left(I^{X}, M_{d}\right.$ *) be the induced fuzzy metric space. If the fuzzy sequence $\left\langle\lambda_{x_{n}}^{\alpha}\right\rangle$ in $I^{X}$ fuzzy converges to $\lambda_{x}^{\alpha}$, then $\left\langle\lambda_{x_{n}}^{\alpha}\right\rangle$ is a fuzzy Cauchy sequence.

Proof. We have by definition, $M_{d}\left(\lambda_{x_{n}}^{\alpha}, \lambda_{x_{m}}^{\alpha}, t+\varepsilon\right) \geq M\left(\lambda_{x_{n}}^{\alpha}, \lambda_{x}^{\alpha}, t\right) *$ $M\left(\lambda_{x}^{\alpha}, \lambda_{x_{m}}^{\alpha}, \varepsilon\right)$ for each $t>0$ and $\varepsilon>0$. Also we have $M_{d}\left(\lambda_{x_{n}}^{\alpha}, \lambda_{x}^{\alpha}, t\right)=$ $\frac{t}{t+d\left(x_{n}, x\right)} \rightarrow 1$ and $M\left(\lambda_{x}^{\alpha}, \lambda_{x_{m}}^{\alpha}, \varepsilon\right)=\frac{\varepsilon}{\varepsilon+d\left(x, x_{m}\right)} \rightarrow 1$. Implies that $M_{d}\left(\lambda_{x_{n}}^{\alpha}, \lambda_{x_{m}}^{\alpha}, t+\right.$ $\varepsilon) \rightarrow 1$ and since $\varepsilon$ is arbitrary small, so $\left\langle\lambda_{x_{n}}^{\alpha}>\right.$ is a fuzzy Cauchy sequence.

Theorem 4.15. A sequence $<\lambda_{x_{n}}^{\alpha}>$ in a fuzzy metric space $\left(I^{X}, M_{d}, *\right)$ is a fuzzy Cauchy sequence if and only if the sequence $\left\langle x_{n}\right\rangle$ is a Cauchy sequence in $(X, d)$.

Proof. Suppose that $\left\langle\lambda_{x_{n}}^{\alpha}\right\rangle$ is a Cauchy sequence, so by definition

$$
\lim _{n, m \rightarrow \infty} M\left(\lambda_{x_{n}}^{\alpha}, \lambda_{x_{m}}^{\alpha}, t\right)=1 \quad \forall t>0 .
$$

$\Longleftrightarrow$ for each small real number $\varepsilon>0$, there exists $K \in N$ such that $1-M\left(\lambda_{x_{n}}^{\alpha}, \lambda_{x_{m}}^{\alpha}, t\right)<\frac{\varepsilon}{\varepsilon+t}$ for all $n>K$.

$\Longleftrightarrow \frac{t}{t+d\left(x_{n}, x_{m}\right)}>1-\frac{\varepsilon}{\varepsilon+t}$ for all $n>K$.

$\Longleftrightarrow d\left(x_{n}, x_{m}\right)<\varepsilon \Longleftrightarrow<x_{n}>$ is a Cauchy sequence.

Remark 4.16. It is obvious that the sequence $\left\langle x_{n}\right\rangle=<1+\frac{1}{2}+\frac{1}{3}+$ $\ldots+\frac{1}{n}>$ is not a Cauchy sequence in the usual metric $(R,||$.$) . Hence, by$ Theorem 4.15 the fuzzy sequence $\left\langle\lambda_{x_{n}}>\right.$ is not a fuzzy Cauchy sequence in $\left(I^{R}, M_{|\cdot|}, *\right)$.

Theorem 4.17. The fuzzy metric space $\left(I^{R}, M_{|\cdot|}, *\right)$ is fuzzy complete. 
Proof. Let $\left(I^{R}, M_{|.|}, *\right)$ be the induced fuzzy metric space and $<\lambda_{x_{n}}>$ be a fuzzy Cauchy sequence in it. Then by Theorem $4.15,\left\langle x_{n}\right\rangle$ is a Cauchy sequence in the complete metric space $(R,||$.$) . Hence \left\langle x_{n}\right\rangle$ converges to some point $x \in R$. Therefore, by Theorem 4.10 we obtain that $<\lambda_{x_{n}}>$ fuzzy converges to $\lambda_{x}$ implies that $\left(I^{R}, M_{|\cdot|}, *\right)$ is fuzzy complete.

Theorem 4.18. The fuzzy metric space $\left(I^{X}, M_{d}, *\right)$ is fuzzy complete if and only if $(X, d)$ is complete.

Proof. Follows from Theorem 4.15 and similar statements of Theorem 4.17.

Theorem 4.19. Let $\left(I^{X}, M_{d}\right.$, *) be the induced fuzzy metric space and let $<\lambda_{x_{n}}>$ be a fuzzy sequence which is fuzzy convergent to $\lambda_{x}$. If $\lambda_{x_{n}} \in$ $B[\mu, r, t]$ for some $\mu \in I^{X}$ and all $n \in N$, then $\lambda_{x} \in B[\mu, r, t]$.

Proof. For any given $\varepsilon>0$ and any $t>0$, we have $M_{d}\left(\mu, \lambda_{x}, t+\varepsilon\right) \geq$ $M_{d}\left(\mu, \lambda_{x_{n}}, t\right) * M_{d}\left(\lambda_{x_{n}}, \lambda_{x}, \varepsilon\right)$. Since $\lambda_{x_{n}} \in B[\mu, r, t]$, so $M_{d}\left(\mu, \lambda_{x_{n}}, t\right) \geq 1-r$. Also $\lambda_{x_{n}} \rightarrow \lambda_{x}$ and $\left(I^{X}, M_{d}, *\right)$ is the induced fuzzy metric space, hence $M_{d}\left(\lambda_{x_{n}}, \lambda_{x}, \varepsilon\right)=M_{d}\left(\lambda_{x}, \lambda_{x_{n}}, \varepsilon\right) \rightarrow 1$. Therefore, $M_{d}\left(\mu, \lambda_{x}, t+\varepsilon\right) \geq(1-$ $r) * 1$. Hence, for a large number $n \in N$, if we choose $\varepsilon=\frac{1}{n}$, then we get $M_{d}\left(\mu, \lambda_{x}, t\right)=\lim _{n \rightarrow \infty} M_{d}\left(\mu, \lambda_{x}, t+\frac{1}{n}\right) \geq 1-r$. Implies that $\lambda_{x} \in B[\mu, r, t]$.

Theorem 4.20. Let $\left(I^{X}, M_{d}\right.$ *) be a complete induced fuzzy metric space and let $B_{1}, B_{2}, \ldots$ be a countable number of fuzzy dense fuzzy open balls in $\left(I^{X}, M_{d}, *\right)$, then $\bigcap_{i=1}^{\infty} B_{i}$ is also fuzzy dense in $\left(I^{X}, M_{d}, *\right)$.

Proof. Let $G_{0}$ be any open ball in $\left(I^{X}, M, *\right)$. Since $B_{1}$ is dense, so $G_{0} \cap B_{1} \neq \phi$, let $\lambda_{1} \in G_{0} \cap B_{1}$. Hence, by Proposition 3.15, there exists $0<r_{1}<1$ and $t_{1}>0$ such that $B\left(\lambda_{1}, r_{1}, t_{1}\right) \subseteq G_{0} \cap B_{1}$. We can take $r_{1}{ }^{\prime}<r_{1}$ and $t_{1}{ }^{\prime}<t_{1}$ such that $B\left[\lambda_{1}, r_{1}{ }^{\prime}, t_{1}{ }^{\prime}\right] \subseteq B\left(\lambda_{1}, r_{1}, t_{1}\right) \subseteq G_{0} \cap B_{1}$. Let $G_{1}=B\left(\lambda_{1}, r_{1}{ }^{\prime}, t_{1}{ }^{\prime}\right)$ and since $B_{2}$ is dense, so $G_{1} \cap B_{2} \neq \phi$, let $\lambda_{2} \in G_{1} \cap B_{2}$. Again by Proposition 3.15, there exists $0<r_{2}<\frac{1}{2}$ and $t_{2}>0$ such that $B\left(\lambda_{2}, r_{2}, t_{2}\right) \subseteq G_{1} \cap B_{2}$. Choosing $r_{2}{ }^{\prime}<r_{2}$ and $t_{2}{ }^{\prime}=$ min. $\left\{t_{2}, \frac{1}{2}\right\}$ such that $B\left[\lambda_{2}, r_{2}{ }^{\prime}, t_{2}{ }^{\prime}\right] \subseteq B\left(\lambda_{2}, r_{2}, t_{2}\right) \subseteq G_{1} \cap B_{2}$, let $G_{2}=B\left(\lambda_{2}, r_{2}{ }^{\prime}, t_{2}{ }^{\prime}\right)$. Continuing in this manner we can find $\lambda_{n} \in G_{n-1} \cap B_{n}$ and $0<r_{n}<\frac{1}{n}$ and $t_{n}>0$ such that $B\left(\lambda_{n}, r_{n}, t_{n}\right) \subseteq G_{n-1} \cap B_{n}$. take $r_{n}{ }^{\prime}<r_{n}$ and $t_{n}{ }^{\prime}=\min .\left\{t_{n}, \frac{1}{n}\right\}$ such that $B\left[\lambda_{n}, r_{n}{ }^{\prime}, t_{n}{ }^{\prime}\right] \subseteq B\left(\lambda_{n}, r_{n}, t_{n}\right) \subseteq G_{n-1} \cap B_{n}$, let $G_{n}=B\left(\lambda_{n}, r_{n}{ }^{\prime}, t_{n}{ }^{\prime}\right)$. 
For any $m>n$ we have $\lambda_{m} \in B\left(\lambda_{n}, r_{n}{ }^{\prime}, t_{n}{ }^{\prime}\right)$. Now for a given $\varepsilon>0$ we can choose $K$ such that $\frac{1}{K}<t$ and $\frac{1}{K}<\varepsilon$. Hence for $m>n>K$, we have $M_{d}\left(\lambda_{n}, \lambda_{m}, t\right) \geq M_{d}\left(\lambda_{n}, \lambda_{m}, \frac{1}{K}\right) \geq 1-\frac{1}{K}>1-\varepsilon$. Hence $\left\langle\lambda_{n}\right\rangle$ is a fuzzy Cauchy sequence. Since $\left(I^{X}, M_{d}, *\right)$ is a complete induced fuzzy metric space, so $\left\langle\lambda_{n}\right\rangle$ fuzzy converges to some fuzzy point $\lambda$. We have $\lambda_{k} \in B\left[\lambda_{n}, r_{n}{ }^{\prime}, t_{n}{ }^{\prime}\right]$ for all $k \geq n$, so by Theorem 4.19 , we obtain that $\lambda \in B\left[\lambda_{n}, r_{n}{ }^{\prime}, t_{n}{ }^{\prime}\right] \subseteq G_{n-1} \cap B_{n}$ for all $n$. Hence, we get

$$
G_{0} \cap\left(\bigcap_{i=1}^{\infty} B_{i}\right)=\phi .
$$

This implies that $\bigcap_{i=1}^{\infty} B_{i}$ is fuzzy dense in $I^{X}$.

\section{Conclusion}

In this paper, we introduced a new type of fuzzy metric space and gave several of its properties. Also we studied some topological properties of this space. The concept of fuzzy convergence and fuzzy Cauchy sequences are investigated and some results on these concepts are obtained. Further, a fuzzy complete space is defined and a connection between a fuzzy complete and a complete metric spaces are discussed.

\section{References}

[1] S. Aytar, "Statistical limit points of sequences of fuzzy numbers", Information Sciences, vol. 165, no. 1-2, pp. 129-138, 2004.

[2] M. Aphane, "On some results of analysis in metric spaces and fuzzy metric spaces", MSc. Thesis, University of South Africa, 2009.

[3] M. K. Chakraborty and T. M. G. Ahsanullah, "Fuzzy topology on fuzzy sets and tolerance topology", Fuzzy Sets and Systems, vol. 45, pp. 103-108, 1992.

[4] C. L. Chang, "Fuzzy topological spaces", Journal of Mathematical Analysis and Applications, vol. 24, pp. 182-190, 1968.

[5] P. Das, "Fuzzy topology on fuzzy sets: product fuzzy topology and fuzzy topological groups", Fuzzy Sets and Systems, vol. 100, pp. 367-372, 1998. 
[6] P. Das and S. K. Ghosal, "Some further results on I-Cauchy sequences and condition (AP)", Computers \& Mathematics with Applications, vol. 59, no. 8, pp. 2597-2600, 2010.

[7] Z. Deng, "Fuzzy Pseudo-Metric Spaces", Journal of Mathematical Analysis and Applications, vol. 86, no. 1, pp. 74-95, 1982.

[8] G. Di Maio and L. D. R. Kočinac, "Statistical convergence in topology", Topology and its Applications, vol. 156, no. 1, pp. 28-45, 2008.

[9] A. George and P. Veeramani, "On some results in fuzzy metric spaces", Fuzzy Sets and Systems, vol. 64, pp. 395-399, 1994.

[10] O. Kaleva and S. Seikkala, "On fuzzy metric spaces", Fuzzy Sets and Systems, vol. 12, pp. 215-229, 1984.

[11] W. J. Pervin, Foundations of General Topology. London: Academic Press, 1964.

[12] R. Srivastava, N. Lal and A. K. Srivastava, "Fuzzy Hausdorff Topological Spaces", Journal of Mathematical Analysis and Applications, vol. 81, no.2, pp. 497-506, 1981.

[13] B. C. Tripathy and S. Debnath, "On generalized difference sequence spaces of fuzzy numbers", Acta Scientiarum Technology, vol. 35, no. 1, pp. 117-121, 2013.

[14] B. C. Tripathy and S. Debnath, " $\gamma$-open sets and $\gamma$-continuous mappings in fuzzy bitopological spaces", Journal of Intelligent and Fuzzy Systems, vol. 24, no. 3, pp. 631-635, 2013.

[15] B. C. Tripathy and S. Debnath, "On fuzzy b-locally open sets in bitopological spaces", Songklanakarin Journal of Science and Technology, vol. 37, no. 1, pp. 93-96, 2015.

[16] B. C. Tripathy and G. C. Ray, "Mixed fuzzy ideal topological spaces", Applied Mathematics and Computations, vol. 220, pp. 602-607, 2013.

[17] B. C. Tripathy and G. C. Ray, "On $\delta$-continuity in mixed fuzzy topological spaces", Boletim da Sociedade Paranaense de Matemática, vol. 32, no. 2, pp. 175-187, 2014.

[18] B. C. Tripathy and A.J. Dutta, "Statistically pre-Cauchy fuzzy realvalued sequences defined by Orlicz function", Proyecciones (Antofagasta), vol. 33, no. 3, pp. 235-243, 2014. 
[19] B. C. Tripathy and G. C. Ray, "Weakly continuous functions on mixed fuzzy topological spaces", Acta Scientiarum Technology, vol. 36, no. 2, pp. 331-335, 2014.

[20] L. A. Zadeh, "Fuzzy Sets", Information and Control, vol. 8, no. 3, pp. 338-353, 1965.

[21] J. Zhang, "The continuity and boundedness of fuzzy linear operators in fuzzy normed space", Journal of Fuzzy Mathematics, vol. 13, no. 3, pp. 519-536, 2005.

\author{
Alias B. Khalaf \\ Department of Mathematics \\ College of Science \\ University of Duhok \\ Kurdistan Region \\ Iraq \\ e-mail: aliasbkhalaf@uod.ac \\ Corresponding author \\ and \\ Maha Waleed \\ Department of Mathematics \\ College of Science \\ University of Duhok \\ Kurdistan Region \\ Iraq \\ e-mail: maha.abdulqader@uod.ac
}

